\title{
DE L'IMMÉDIATEMENT DONNÉ AU "DÉTOUR DE L'EXPÉRIENCE MYSTIQUE”. REMARQUES SUR L'UNITÉ DE LA MÉTHODE INTUITIVE CHEZ BERGSON $^{1}$
}

\author{
Anthony Feneuil (Université de Genève) ${ }^{2}$ \\ anthony.feneuil@unige.ch
}

Résumé: Bergson avait-il prévu dès 1903, dans un article qui annonçait la possibilité de passer par approfondissement de l'intuition du moi à celle d'une "éternité de vie", la suite de son œuvre, et en particulier son grand livre de 1932, Les Deux Sources de la morale et de la religion? Oui, si rien, dans sa méthode philosophique intuitive, n'a changé en trente ans. A la suite de Jean Nabert (1941), cet article veut montrer que la méthode philosophique bergsonienne subit un changement majeur en 1932, contrainte d'abandonner l'immédiateté qui la caractérisait et découvrant la médiation.

Mots-clés: Bergson; intuition; immédiateté; médiation; mysticism.

Le cheminement de Bergson, depuis l'Essai sur les données immédiates de la conscience (1889) jusqu'aux Deux Sources de la morale et de la religion (1932), était imprévisible: tous les grands lecteurs de Bergson, pourtant attentifs à chercher l'unité de l'œuvre, s'accordent à le dire. Comment pourraient-ils faire autrement? Ce serait aller trop explicitement

\footnotetext{
${ }^{1}$ Recebido: 15-03-2012/Aprovado: 28-05-2012/Publicado on-line: 15-09-2012.

${ }^{2}$ Anthony Feneuil é doutorando e Assistente de ensino da Université de Genève, Genebra, Suiça.
} 
contre la pensée du maître ${ }^{3}$. Et pourtant, il était prévu. Par Bergson lui-même, dans l'un de ses plus célèbres articles, paru en 1903 dans la Revue de métaphysique et de morale, "Introduction à la métaphysique”. Qu'était-il prévu exactement? Que la méthode philosophique découverte par Bergson, l'intuition (BERGSON 1903, 181), permettrait de passer, "par un effort" (BERGSON 1903, 210), de la connaissance du "moi qui dure" (BERGSON 1903, 182) à celle d'une "éternité de vie" (BERGSON 1903, 210) qui constitue à n'en pas douter la préfiguration du Dieu enfin accessible à la philosophie des Deux Sources. Comment le permettrait-elle?

[...] de même qu'une conscience à base de couleur, qui sympathiserait intérieurement avec l'orangé au lieu de le percevoir extérieurement, se sentirait prise entre du rouge et du jaune, pressentirait même peut-être, au-dessous de cette dernière couleur, tout un spectre en lequel se prolonge naturellement la continuité qui va du rouge au jaune (BERGSON 1903, 210).

Tout n'était-il pas dit du mouvement "d'approfondissement" de la durée qui constitue, pour Camille Riquier dans sa belle Archéologie de Bergson, le mouvement même de l'œuvre Bergsonienne (RIQUIER 2009, 105-117)? Un grand pas était en tout cas déjà fait, et il fallait vraiment que la mauvaise conscience à l'égard de Bergson fût grande pour qu'aucun de ses commentateurs n'entreprît de décrire l'évolution de son ouvre après 1903 comme la réalisation d'un plan établi à l'avance. On s'en est tenu à la présentation que Bergson, mais avant d'écrire

\footnotetext{
3 "Comment concevez-vous, par exemple, la grande œuvre dramatique de demain?" Je me rappellerai toujours la surprise de mon interlocuteur quand je lui répondis: "Si je savais ce que sera la grande œuvre dramatique de demain, je la ferais.” (BERGSON 1930, 110)
} 
son dernier livre, donnait de sa propre pensée ${ }^{4}$. On a cherché l'unité de l'œuvre, plutôt que dans un plan préétabli, dans l'unité de l'impulsion initiale, acte devenu méthode, dans l'intuition ${ }^{5}$. Mais peut-on vraiment, une fois située l'unité du bergsonisme dans cette méthode intuitive si profondément réfléchie en 1903, échapper au doute que le bergsonisme serait déjà tout entier dans l'Introduction à la métaphysique, et que Bergson n'aurait fait ensuite qu'en suivre les grandes lignes, si bien que tout le bergsonisme serait une contradiction performative? A moins que l'apparente proximité entre ce texte de 1903 et celui des Deux Sources ne relevât de l'illusion rétrospective, mais alors il faudrait montrer en quoi.

En 1941, l'année même de la mort de Bergson, Jean Nabert publie un article trop peu cité ${ }^{6}$, qui met précisément en cause l'unité de la théorie l'intuition dans le bergsonisme, et pointe au contraire un tournant qui s'opérerait dans Les Deux Sources, ou du moins une tension qui s'y révélerait, quoiqu'à bas bruit et sans que toutes les conséquences en soient explicitement tirées. Après avoir voulu fonder sa philosophie, et l'espérance en la philosophie en générale, sur la méthode intuitive ${ }^{7}$, et fondé la validité de la

\footnotetext{
${ }^{4}$ Henri Bergson, "Introduction I", La Pensée et le mouvant, Paris, PUF, 2010, p. 20.

${ }^{5}$ Citons, parmi les plus importants, Gilles Deleuze d'abord, et plus récemment Camille Riquier et Frédéric Worms: "De fait, on peut d'abord dire, avec Bergson lui-même dans le résumé rétrospectif qu'il donne de son œuvre dans La pensée et le mouvant que, malgré leur diversité, tous ses livres reprennent avant tout la surprise initiale dans son contenu le plus propre (l'écart entre le temps ou plutôt la durée et l'espace, entre l'acte et ses résultats) et dans sa portée méthodologique essentielle, qui permet d'en parler comme d'une intuition, ou de parler de l'intuition comme d'une méthode" (Frédéric Worms, Bergson ou les deux sens de la vie, Paris, PUF, 2004, p. 347).

${ }^{6}$ Mentionné par Frédéric Worms dans son introduction à l'édition critique des Deux Sources.

${ }^{7}$ L'insistance de Bergson sur la différence entre l'intuition et un "instinct vague" ou une pensée imprécise a été soulignée par la plupart de ses commentateurs. Voir BERGSON 1922, 95. Cf. DELEUZE 1998, 1: "L'intuition n'est pas un sentiment ni une inspiration, une sympathie confuse, mais une méthode élaborée, et même une des méthodes les plus élaborées de la philosoCont.
} 
méthode intuitive sur son caractère direct et immédiat, sa capacité à nous faire suivre "le réel dans toutes ses sinuosités" et à ôter tout "jeu” (BERGSON 1907, 362) entre l'esprit et les choses, Bergson aurait finalement accepté, dans son dernier grand livre, de perdre le contact et d'en passer par "le détour de l'expérience mystique" (NABERT 1941, 351). D'introduire ainsi, avec la mystique ${ }^{8}$, ce qui jusque là avait été consciemment et résolument exclu de la philosophie: la médiation.

Un mouvement traverse le bergsonisme: il commence avec la découverte de la durée, qu'expose l'Essai sur les données immédiates de la conscience. Cette découverte en entraîne d'autres, mais d'abord celle de la puissance potentielle d'une philosophie qui penserait "en durée" , autrement dit qui chercherait à toujours saisir l'immédiatement perceptible et par conséquent le réellement donné. La philosophie n'est plus un procédé de construction de modèles théoriques, mais un acte de dévoilement du donné dans la conscience, habituellement dissimulé par le langage et la pensée naturelle (BERGSON 1889, VII). Dans cet immédiat dévoilé, c'est la chose elle-même qui est saisie. D'abord la conscience qui dure, dans l'Essai sur les données immédiates de la conscience. Ensuite, dans Matière et mémoire, avec l'examen de la perception, la relation de la conscience au corps. Et là, probablement, une digue se rompt et une nouvelle découverte a lieu, relative encore à la puissance de la philosophie. Avec la saisie du lien entre la perception et

\footnotetext{
phie."

8 "Si j'apporte, dans ces pages, quelque chose de nouveau, c'est cela. Je tente d'introduire la mystique en philosophie, comme procédé de recherche philosophique" (Bergson apud J. Chevalier, CHEVALIER 1959, 152).

9 "Penser intuitivement est penser en durée." (BERGSON 1922, 30)
} 
l'utilité, et par là du fameux "tournant de l'expérience" (BERGSON 1896, 206), c'est-à-dire de la conscience humaine comme simple dérivé d'une forme de conscience plus large, l'ambition peut naître d'étendre ce mode de pensée que Bergson appelle de plus en plus facilement "intuition”, jusqu'aux racines non seulement de la conscience individuelle, mais de l'espèce et de la vie, dans le mouvement même par lequel elle crée la matière: ce sera L'Évolution créatrice ${ }^{10}$.

Mais ce mouvement d'approfondissement de la découverte initiale est lui-même, et dès le début, en tension. Car à mesure que la philosophie de Bergson découvre sa puissance propre, puissance de résolution des problèmes et de saisie de l'absolu non seulement du moi, mais du monde, elle découvre aussi la nécessité de s'approprier des connaissances qui lui sont extérieures. Ce sont, dans l'Essai, les données de la psychologie empirique. Dans Matière et Mémoire, de la psychopathologie. Dans L'évolution créatrice, où ce travail d'appropriation va le plus loin ${ }^{11}$, les données de la biologie. L'intuition n'est pas un relâchement solipsiste du moi sur lui-même, mais un effort impliquant le recours à des données objectives:

Car on n'obtient pas de la réalité une intuition, c'est-à-dire une sympathie spirituelle avec ce qu'elle a de plus intérieur, si l'on n'a pas gagné sa confiance par une longue camaraderie avec ses manifestations superficielles. Et il ne s'agit pas simplement de s'assimiler les faits marquants; il en faut accumuler et fondre ensemble une si énorme masse qu'on soit assuré, dans cette fusion, de neutraliser les

\footnotetext{
10 Pour une étude poussée de l'évolution de l'œuvre de Bergson comme mouvement d'approfondissement, on se reportera de nouveau à RIQUIER 2009, Deuxième partie.

${ }^{11}$ L'édition critique remarquable d'Arnaud François permet de mesurer l'ampleur de la documentation bergsonienne pour cet ouvrage.
} 
unes par les autres toutes les idées préconçues et prématurées que les observateurs ont pu déposer, à leur insu, au fond de leurs observations (BERGSON 1903, 226).

Ainsi l'intuition suppose-t-elle le travail de l'intelligence, et en particulier l'accumulation des données "superficielles". Que faut-il entendre par là? J'utilise le terme de "données" parce qu'il est courant, et notamment lorsqu'il est question de sciences positives. Pourtant, il convient mal à ce dont il est question. Pour Bergson, les sciences fournissent, plutôt que de véritables données, des vues prises sur les choses par notre intelligence. Or l'intelligence ${ }^{12}$, parce qu'elle est une fonction biologique des vertébrés que nous sommes, est orientée vers l'action utile, et non vers la spéculation. Par conséquent, elle néglige, dans la chose qu'elle prend pour objet, tout ce sur quoi l'on ne saurait avoir prise, et qui par conséquent nous serait inutile: ce qu'elle a de vivant, de créateur, et que nous ne pouvons maîtriser comme nous maîtrisons l'inerte. Comment ces savoirs intellectuels accumulés pourraient-ils mener à l'intuition, c'est-à-dire au contraire à une connaissance libérée de la contrainte de l'action $^{13}$ et qui,

\footnotetext{
${ }^{12}$ Il n'est pas nécessaire d'entrer ici dans le détail de l'évolution de la théorie bergsonienne de l'intelligence, mais notons que si les productions de l'intelligence semblent être pour Bergson, en 1903, purement conventionnelles ("symboliques"), elles acquièrent progressivement, et en tout cas dans L'Évolution créatrice, une portée réaliste. Sinon dans leur détail, du moins dans leur organisation générale, les lois scientifiques touchent à l'absolu: non qu'elles expriment l'ensemble de la réalité, mais son côté matériel (BERGSON 1907, 199-200). C'est que le mouvement de l'intelligence est dans cet ouvrage replacé par Bergson dans le mouvement général de la création, et sa tendance à ne saisir que de l'espace et à évacuer le changement, interprétée comme le corollaire spécifiquement humain du mouvement général de retombée de l'élan créateur, générateur de la matière (BERGSON 1907, 246).

13 "Attelés, comme des boufs de labour, à une lourde tâche, nous sentons le jeu de nos muscles et de nos articulations, le poids de la charrue et la résistance du sol: agir et se savoir agir, entrer en contact avec la réalité et même la vivre, mais dans la mesure seulement où elle intéresse l'œuvre qui s'accomplit et le sillon qui se creuse, voilà la fonction de l'intelligence humaine". (BERGSON 1907, 192)
} 
pour cette raison même, laisse la chose se donner comme elle est plutôt que comme nous avons besoin de la voir?

En réalité, il n'y a pas de continuité entre les données scientifiques et l'intuition. Aucune donnée positive extérieure à l'intuition philosophique ne saurait, du moins encore en 1907, se substituer à elle ou même simplement combler ses éventuelles lacunes. C'est de manière toute négative, par leurs déficiences et leur hétérogénéité au temps véritable et à la création, que ces données peuvent susciter et orienter l'intuition. Dialectiquement ${ }^{14}$, en somme:

[...] elle [l'intuition] utilisera le mécanisme même de l'intelligence à montrer comment les cadres intellectuels ne trouvent plus ici leur exacte application, et d'autre part, par son travail propre, elle nous suggérera tout au moins le sentiment vague de ce qu'il faut mettre à la place des cadres intellectuels. (BERGSON 1907, 178-9)

\section{Une philosophie digne de ce nom ne saurait donc se passer} des données positives extérieures, celles que les sciences accumulent et organisent selon les principes de l'intelligence: faits de la psychologie expérimentale, de la biologie ou encore, dans Les Deux Sources, de la sociologie. C'est pourquoi

\footnotetext{
${ }^{14}$ Le rapport de la science moderne à l'intuition est l'exemple de cette dialectique. La science moderne est une véritable avancée dans le développement de l'intelligence, parce qu'elle cesse de chercher à formuler l'essence des choses, et s'oriente désormais vers les lois de leur mouvement. C'est précisément pour cette raison qu'avec elle se manifeste négativement l'hétérogénéité radicale entre le mouvement et l'intelligence: "La science moderne date du jour où l'on érigea la mobilité en réalité indépendante" (BERGSON 1903, 217). "Ce qu'on étudie [dans la science moderne], ce sont les choses qui s'écoulent. Il est vrai que sur la réalité qui coule on se borne à prendre les instantanés. Mais justement pour cette raison, la connaissance scientifique devrait en appeler une autre, qui la complétât." (TROTIGNON 1968, 344). Lorsque l'on décrète, comme dans la science antique, que le mouvement n'est pas objet de connaissance, l'esprit peut se tenir tranquille et se satisfaire de la compréhension intellectuelle du monde. Mais dès lors que la science prétend s'intéresser au mouvement, et qu'elle n'en étudie que des instantanés, de telle sorte que "si tous les mouvements de l'univers se produisaient deux ou trois fois plus vite, il n'y aurait rien à modifier à nos formules, ni aux nombres que nous y faisons entrer" (BERGSON 1889, 87), la contradiction prend "un tour intolérable et absurde" (TROTIGNON 1968, 340).
} 
le philosophe doit être "toujours prêt, quel que soit son âge, à se refaire étudiant" (BERGSON 1922b, 72-73). Le risque serait grand, sinon, que la philosophie se replie sur elle-même, sans savoir dans quelle direction orienter son effort. Et cependant, l'aide que ces données lui apportent reste irrémédiablement extérieure. Elles fonctionnent comme des aiguillons de l'intuition, sans jamais la pénétrer: ainsi la comparaison des descriptions entomologiques de "diverses formes du même instinct dans diverses espèces d'Hyménoptères", par lequel ces espèces paralysent leurs proies sans les tuer tout de suite, montre d'abord que le progrès des connaissances, le raffinement dans la compréhension mécaniste des étapes par lesquelles l'hyménoptère procède dans son entreprise meurtrière, a pour effet paradoxal de rendre plus difficile la compréhension de la genèse de cet instinct. Mieux l'intelligence dévoile la complexité d'un phénomène, plus il est délicat de rendre compte intellectuellement de son origine ${ }^{15}$. Mais précisément, le dévoilement de cette insuffisance nous renvoie à nous-même: n'expérimentons pas en nous quelque chose du genre de ce dont la science est par principe incapable de rendre compte, et dont elle ne doit d'ailleurs pas chercher à rendre compte, mais dont l'absence est rendue criante par l'insuffisance de ses explications?

Mais il n'en serait plus de même si l'on supposait entre le Sphex et sa victime une sympathie (au sens étymologique du mot) qui le renseignât du dedans, pour ainsi dire, sur la vulnérabilité de la Chenille. [...] Certes, une théorie scientifique ne peut faire appel à des consi-

\footnotetext{
15 "À supposer que, dans la suite des temps, le Sphex soit arrivé à reconnaître un à un, par tâtonnement, les points de sa victime qu'il faut piquer pour l'immobiliser, et le traitement spécial qu'il faut infliger au cerveau pour que la paralysie vienne sans entrainer la mort, comment supposer que les éléments si spéciaux d'une connaissance si précise se soient transmis régulièrement, un à un, par hérédité?" (BERGSON 1907, 174)
} 
dérations de ce genre. Elle ne doit pas mettre l'action avant l'organisation, la sympathie avant la perception et la connaissance. [...] Dans des phénomènes de sentiment, dans des sympathies et des antipathies irréfléchies, nous expérimentons en nous- mêmes, sous une forme bien plus vague, et trop pénétrée aussi d'intelligence, quelque chose de ce qui doit se passer dans la conscience d'un insecte agissant par instinct. (BERGSON 1907, 175-6)

Le raisonnement est ici, pour les besoins de l'argumentation, simplifié à l'extrême: il supposerait de clarifier le rapport entre intuition et instinct, et du coup de revenir plus généralement sur la question des rapports entre espèces, qui chez Bergson fait partie intégrante de la théorie de la connaissance. Reste l'essentiel pour nous: le rapport entre données scientifiques et intuition. Ce rapport n'est pas direct, mais dialectique: la philosophie s'attache à comprendre les sciences pour en marquer les lacunes qui la renvoient à elle-même, en l'orientant toutefois dans des directions vers lesquelles elle ne serait pas allée seule. Il n'y a donc pas de pénétration des données scientifiques dans la philosophie intuitive. En revanche, la philosophie peut, elle, éclairer en retour les données scientifiques, en les replaçant dans le mouvement de leur genèse, qu'elle est seule capable de saisir, l'intelligence étant par principe rétive à la création. La philosophie s'introduit dans les sciences, jusqu'à éventuellement les réformer ${ }^{16}$.

Bref il y a bien, dans le bergsonisme, et parallèlement au mouvement centripète d'approfondissement de l'intuition initiale, un mouvement centrifuge qui pousse le philosophe au contact des faits scientifiques les plus variés

\footnotetext{
16 "Nous disions qu'il faut amener la philosophie à une précision plus haute, la mettre à même de résoudre des problèmes plus spéciaux, faire d'elle l'auxiliaire et, s'il est besoin, la réformatrice de la science positive." (BERGSON 1922b, 70)
} 
et les plus neufs. Cependant, au moins jusqu'en 1907 et même dans certains passages du livre de 1932, le mouvement centripète l'emporte sur le mouvement centrifuge, jusqu'à l'intégrer comme un de ses moments: les faits orientent le questionnement du philosophe, le philosophe donne leur sens aux faits, mais jamais l'intuition philosophique n'est médiatisée par des faits qui lui seraient extérieurs:

[...] on voyait le philosophe interroger les faits, percer leur enveloppe, pénétrer au-dessous des concepts ou de l'intelligence sociale, obtenir de l'expérience qu'elle laissât lire sa signification intrinsèque. Bref, le retour à l'immédiat, l'intuition, donnaient la réalité véritable. (NABERT 1941, 356)

Or le dernier livre de Bergson, en 1932, s'il poursuit bien cette tendance de la philosophie à chercher hors d'ellemême sa direction, la pervertit tout autant. Car le sens de ce que Bergson appelle alors, pour caractériser l'essentiel de son dernier livre, l'introduction de la mystique en philosophie, "comme méthode philosophique" (BERGSON 1933, 1507), est tout autre que celui de l'appropriation philosophique des données de la biologie dans L'Évolution créatrice. Il ne s'agit plus seulement d'une annexion par la philosophie de données qui lui sont extérieures. Il ne s'agit plus seulement, en d'autres termes, de se laisser indiquer négativement le chemin de l'immédiatement donné en soi, pour retrouver ensuite, à partir de là, le sens des données extérieures. Ou plutôt, ce chemin de soi vers l'autre dans la philosophie, cette annexion des données extérieures n'est plus possible que par un "détour", par la reconnaissance de la primauté, pour accéder à l'immédiat par excellence, pour accéder à l'identité de la conscience avec son principe, d'une expérience non philosophique mais qui n'est pas dépour- 
vue en elle-même de sens positif, et que le philosophe peut seulement tenter de serrer au plus près, mais à laquelle il doit lui-même renoncer dans sa plénitude.

L'examen de tous les signes qui, dans le parcours de Bergson depuis L'Évolution créatrice jusqu'au Deux Sources de la morale et de la religion, témoignent de cette évolution, demanderait une étude à part. Contentons-nous ici d'en marquer deux des plus nets symptômes.

Le premier est la conférence de 1911 sur "L'intuition philosophique" ${ }^{17}$, qui introduit, à l'occasion d'une réflexion sur l'histoire de la philosophie, une nouveauté dans la théorie de l'intuition souvent passée inaperçue. Considérant la suite des grandes doctrines philosophiques, Bergson introduit, dans sa compréhension de la philosophie, un élément surprenant: un écart entre la doctrine philosophique considérée comme une "totalité organique" et ce qui en fait l'unité la plus propre, l'intuition à son origine. Toute philosophie est alors constituée de trois strates: l'intuition à son origine (son âme), la totalité organique qu'elle constitue (son corps, saisi tel qu'en lui-même), les thèses et les concepts qui la composent (son corps, saisi comme la mécanique peut saisir le corps vivant):

Ainsi les diverses parties du système s'entrepénètrent, comme chez un être vivant. Mais, comme je le disais au début, le spectacle de cette pénétration réciproque nous donne sans doute une idée plus juste du corps de la doctrine; il ne nous en fait pas encore atteindre l'âme. (BERGSON 1911a, 129)

Cette innocente déclaration est en fait très problématique:

\footnotetext{
${ }^{17}$ Je renvoie à l'édition critique de ce texte, que j'ai établie avec Ghislain Waterlot dans l'édition des PUF en 2009.
} 
on serait plutôt tenté d'identifier la philosophie, non certes comme ensemble de thèses, mais comme totalité organique, à l'expérience de la durée dont elle veut rendre compte, c'est-à-dire au niveau de l'intuition telle qu'elle est définie en 1903. Pourquoi Bergson ajoute-t-il un niveau supplémentaire, qui semble justement situer l'intuition hors $d u$ temps, hors de la durée effectivement vécue par le philosophe?

Plus nous remontons vers cette intuition originelle, plus nous comprenons que, si Spinoza avait vécu avant Descartes, il aurait sans doute écrit autre chose que ce qu'il a écrit, mais que, Spinoza vivant et écrivant, nous étions sûrs d'avoir le spinozisme tout de même (BERGSON 1911a, 124)

Ne doit-on pas voir là une première secousse dans la théorie de l'intuition telle qu'elle est formulée en 1903? Car en 1911, le niveau de la durée pure, du flux continu de la conscience, auquel correspond celui de la philosophie comme totalité organique n'est plus originaire: il n'est plus entièrement à lui-même sa propre vérité. Il découle d'autre chose, d'une intuition plus simple encore, d'une âme dont il n'est que le corps, et à laquelle il n'est pas évident de remonter. Certes, on pourrait dire que cette idée d'une intuition absolument simple, et par là plus simple que la continuité de changement dont elle est à l'origine, rejoint en partie celle de la durée infiniment contractée de l'éternité de vie dont nous étions partis. A ce moment là, l'écart entre la durée vécue par Spinoza et son intuition fondamentale serait exactement celui que suppose l'idée d'une transcendance de soi-même par soi-même ${ }^{18}$, déjà envisagée en 1903. Et d'ailleurs, il est vrai que Bergson envisage la possibilité sinon de rejoindre du moins d'approcher

18 “nous nous transcendons nous-mêmes”. (BERGSON 1903, 210) 
cette intuition simple par un effort d'approfondissement de la compréhension de la totalité organique de la doctrine. Et cependant, on voit bien que, dans le détail, il est assez difficile de faire en sorte que les deux théories se recoupent tout à fait, parce que rien dans la conférence de 1903 ne laisse penser que les durées moins contractées que nous sommes découlent de la durée la plus contractée qu'est l'éternité de $v_{i e}{ }^{19}$. En outre, identifier l'intuition simple au principe de chaque doctrine à la durée dans son maximum de contraction obligerait à affirmer que tous les philosophes expérimenteraient en fait la même chose, l'éternité de vie, la durée contractée au maximum: mais pourquoi, alors, Spinoza écrivant avant Descartes aurait-il été Spinoza, et non pas Descartes ${ }^{20}$ ? Il est vrai que les deux conférences n'ont pas le même objet: tous les rapprochements et les contrastes entre elles ne peuvent manquer d'avoir quelque chose d'artificiel, dès lors qu'ils deviennent trop précis. C'est pourquoi je me contente de parler ici de symptômes d'une évolution, sans chercher à déterminer trop précisément cette évolution à partir de ces deux seules conférences - le dossier demanderait à être repris et approfondi. Cette réserve importante émise, il reste clair que la conférence de 1911 introduit des éléments qui bousculent en partie les attentes d'un lecteur habitué aux concepts bergsoniens et à leur agencement jusque là ${ }^{21}$.

\footnotetext{
${ }^{19}$ C'est déjà quelque peu différent dans la "perception du changement", puisque l'éternité de vie est identifiée au "principe". (BERGSON 1911b, 176)

${ }^{20} \mathrm{Il}$ y a ici une différence - peut-être réductible, le travail reste à faire - entre ce texte et celui de Bergson (1907, 239): "L'intuition, si elle pouvait se prolonger au-delà de quelques instants, n'assurerait pas seulement l'accord du philosophe avec sa propre pensée, mais encore celui de tous les philosophes entre eux. Telle qu'elle existe, fuyante et incomplète, elle est, dans chaque système, ce qui vaut mieux que le système, et ce qui lui survit."

${ }^{21}$ Cette conférence est également à d'autres égards anticipatrice des plus grandes nouveautés des Cont.
} 
Un autre texte, plus tardif de onze ans (1922) et repris lui aussi en 1934 dans La Pensée et le mouvant, constitue un deuxième symptôme, plus décisif encore. Il est d'autant plus frappant qu'il reprend l'image, cruciale dans le texte de 1903, de l'orangé, pour littéralement la renverser. L'absence, à notre connaissance, de tout commentaire de ce renversement est également très étonnante, parce qu'il s'agit d'une image couramment utilisée dans les présentations du bergsonisme ${ }^{22}$, et à laquelle Bergson semble luimême beaucoup tenir (il souligne d'ailleurs, dans l'édition de La Pensée et le mouvant, sa reprise à l'identique dans Les Deux Sources). En 1903, l'image de l'orangé était utilisée pour souligner le caractère dynamique et le potentiel d'approfondissement de l'intuition de la durée, déjà riche en elle-même d'une diversité imperceptible au premier abord, comme l'orangé est riche du rouge, du jaune et de tout le spectre des couleurs. En 1922, la même image est utilisée mais exactement à contre-emploi:

Prenons une couleur telle que l'orangé. Comme nous connaissons en outre le rouge et le jaune, nous pouvons considérer l'orangé comme jaune en un sens, rouge dans l'autre, et dire que c'est un composé de jaune et de rouge. Mais supposez que, l'orangé existant tel qu'il est, ni le jaune ni le rouge n'eussent encore paru dans le monde: l'orangé serait-il déjà composé de ces deux couleurs? Evidemment non. (BERGSON 1922a, 18)23

Bien sûr, le contexte n'est pas le même qu'en 1903: l'image ne vise pas à éclairer la nature de l'intuition de la

\footnotetext{
Deux Sources, en particulier de la conception de la personne (voir FENEUIL 2011, 70 sq).

${ }^{22}$ Par exemple dans la notice Henri Bergson du Stanford Encyclopedia of Philosophy (LAWLOR \& MOULARD 2012).

${ }^{23}$ L'exemple est donc repris à l'identique dans Les Deux Sources (BERGSON 1932, 313): "Imaginons que l'orangé soit la seule couleur qui ait encore paru dans le monde: serait-il déjà composé de jaune et de rouge? Évidemment non.”
} 
durée, mais le "mouvement rétrograde du vrai", c'est-à-dire la tendance de l'intelligence à rejeter dans le passé, sous forme de possibilités, ce qui advient, et du coup à transformer rétrospectivement ce qui était simple en un complexe d'éléments ${ }^{24}$. Et en même temps, comment ne pas voir que cette critique du mouvement rétrospectif de la pensée, réemployant l'image du texte de 1903, pourrait aussi s'appliquer à lui? Car on doit se demander dans quelle mesure l'intuition de "l'éternité de vie" était effectivement contenue dans celle du moi qui dure. Ce que le philosophe prétendait tirer de la pure immédiateté de l'intuition philosophique n'y avait-il pas été subrepticement introduit de l'extérieur? Ce qui était compris comme auto-transcendance n'était-il pas en vérité réponse à une transcendance première? Jusqu'où Bergson, reprenant et renversant, vingt ans après, cette image tirée d'un de ses plus célèbres articles, a-til effectivement voulu se critiquer? Voilà qui est indécidable:

\footnotetext{
${ }^{24}$ Le premier usage de cette image par Bergson, repéré par Ghislain Waterlot et Frédéric Keck dans leur édition critique des Deux Sources (p. 499, note 82), est oral, lors d'une séance de la société française de philosophie en 1901. Il se fait dans un contexte plus proche de celui de 1922, même s'il n'est évidemment pas question encore de mouvement rétrograde du vrai. On retrouve la question de la simplicité et de la composition, et de l'irréductibilité de l'orangé au rouge et au jaune: "Quand vous aurez défini quatre positions, choisies le long de la série continue des sens qu'on peut donner à un terme métaphysique, ne croyez pas que vous arriverez, en composant ces sens entre eux, à reproduire les idées intermédiaires. Si ces idées sont vraiment philosophiques et suggestives, ce sont des idées simples. Celui qui ne connaîtrait que le jaune et le rouge n'arriverait jamais à imaginer l'orangé, quoiqu'il soit facile, en voyant l'orangé, d'y retrouver du jaune et du rouge. Ainsi il ne faut pas croire qu'en définissant quelques-uns des points de vue possibles, sur un objet de la recherche philosophique, on donne des indications utiles pour la représentation des points de vue intermédiaires" (BERGSON 1901, 942). Cependant, par rapport à l'occurrence de 1922, l'exemple est pris par l'autre bout: celui de l'impossibilité de déduire l'orangé du rouge et du jaune, et de celle de découvrir dans l'orangé le rouge et le jaune. En sorte qu'il est très difficile de savoir si ce premier usage de l'exemple s'oppose ou non à celui de 1903, parce qu'il ne donne pas d'indication certaine sur la conception de l'orangé qu'il porte. En effet, le terme « retrouver » est ambigu, et il peut être interprété aussi bien selon la conception de 1903 (il est possible, en creusant l'orangé, d'arriver au rouge et au jaune), que selon celle de 1922 (comme nous connaissons déjà le rouge et le jaune, il est possible de les retrouver dans l'orangé).
} 
peut-être a-t-il tout simplement changé d'avis sur l'orangé et sa perception ${ }^{25}$. Il n'empêche que cette autocritique est comme confirmée et amplifiée par les Deux Sources qui, en 1932, ne réalisent la prédiction de 1903 qu'en l'infirmant bien plus encore: non, l'éternité de vie n'était pas à chercher dans la philosophie, et la philosophie elle-même, pour tâcher d'en comprendre quelque chose, devait la chercher ailleurs. Où cela?

[...] il suffirait de prendre le mysticisme à l'état pur, dégagé des visions, des allégories, des formules par lesquelles il s'exprime, pour en faire un auxiliaire puissant de la recherche philosophique. (BERGSON 1932, 266)

C'est ainsi, par la notion d'auxiliaire, que Bergson fait entrer la médiation en philosophie. La mystique, en effet, auxiliaire de la philosophie, n'entretient pas avec elle le même rapport que, dans L'Évolution créatrice, la biologie. Alors que la biologie se contente de soutenir - et dialectiquement, comme on l'a vu - l'intuition philosophique, lui restant par conséquent toujours extérieure, l'intuition mystique prolonge l'intuition philosophique ${ }^{26}$. Elle la prolonge:

\footnotetext{
${ }^{25}$ Et cependant, comment ne pas voir l'ironie de Bergson à l'égard de son propre texte de 1903, qu'il reprend presque textuellement en 1932 mais pour lui attribuer le statut d'une simple "fantaisie", et pour encore une fois en renverser le sens, et affirmer que ce qui se présentait alors comme un processus d'approfondissement du déjà là était en réalité déjà un processus de sortie de soi dans la création: "et si l'on supposait, par un jeu de fantaisie, que le jaune et le rouge ont surgi d'une que le jaune et le rouge ont surgi d'une intensification de l'orangé, on aurait un exemple très simple de ce que nous appelons la croissance en forme de gerbe" (BERGSON 1932, 313, je souligne).

26 “[...] si l'intuition adossée à la science est susceptible d'être prolongée, ce ne peut être que par l'intuition mystique. De fait, les conclusions que nous venons de présenter complètent naturellement, quoique non pas nécessairement, celles de nos précédents travaux" (BERGSON 1932, 272). Avant 1932 c'est, au contraire, la philosophie qui est l'auxiliaire" (BERGSON 1922b, 70) de la science, et qui, dans L'Évolution créatrice, en constitue le prolongement (BERGSON 1907, 369). La permutation des termes est presque systématique: non seulement l'intuition mystique n'est pas à l'intuition philosophique ce que sont les données de la science à la philosophie, mais on peut dire inversement que l'intuition mystique est à l'intuition philosophique ce que la philosophie est à la science: son auxiliaire, et sa réformatrice.
} 
autrement dit, sur ce terrain du "problème de l'existence et de la nature de Dieu" (BERGSON 1932, 255), où la philosophie est incapable d'aller seule, elle s'y substitue, et destitue le philosophe de la position qu'il avait toujours revendiquée, celle de l'immédiateté, de la "vision directe" (BERGSON 1922b, 42). C'est désormais le mystique et non le philosophe, qui se trouve en communication directe avec ce dont il est question. Le philosophe, lui, n'a plus qu'à accepter sa médiation pour qu'il lui livre, à force d'interrogations, le "secret de la création" 27.

Certes, Bergson est prudent. Dans ces pages très denses des Deux Sources, il prend bien soin d'encadrer étroitement l'introduction de la médiation mystique dans ce domaine de l'immédiat qu'est la philosophie. D'une part, il l'encadre de l'extérieur, en prenant soin d'insister sur le fait que le mysticisme, pour posséder une valeur philosophique, doit être reconnu par la philosophie. En effet, celle-ci doit en établir la possibilité a priori:

Nous reconnaissons pourtant que l'expérience mystique, laissée à elle-même, ne peut apporter au philosophe la certitude définitive. Elle ne serait tout à fait convaincante que si celui-ci était arrivé par une autre voie, telle que l'expérience sensible et le raisonnement fondé sur elle, à envisager comme vraisemblable l'existence d'une expérience privilégiée, par laquelle l'homme entrerait en communication avec un principe transcendant. (BERGSON 1932, 263)

D'autre part la philosophie s'arroge, cette fois à l'intérieur même de l'expérience mystique, le droit de séparer le bon grain de l'ivraie, au nom justement de son intuition à elle. Ce processus d'épuration ${ }^{28}$ de la mystique n'est pas sans

\footnotetext{
27 “il livrerait à qui saurait l'interroger le secret de la création." (BERGSON 1932, 248)

${ }^{28}$ Là aussi, il s'agit d'un terme que Bergson utilisait pour caractériser le rapport de la philosophie Cont.
} 
faire courir un risque au philosophe: le risque d'étouffer le discours mystique et de parler à sa place, en plaquant sur lui ses préconceptions ${ }^{29}$. Celui, en d'autres termes, de manquer la médiation. Mais si Bergson prend ce risque, c'est sans doute qu'il sait l'audace de son geste, et la tension qu'il introduit dans le sein même de la philosophie, donc aussi la nécessité de le circonscrire très rigoureusement. Il n'en reste pas moins qu'au cour même de la philosophie, et dans les limites peut-être étroites que nous avons dites, est introduite une intuition étrangère à l'intuition philosophique, et qui cependant en constitue un prolongement en lui-même positif ${ }^{30}$.

Dans cette enceinte de confinement, c'est la connaissance philosophique elle-même qui est redéfinie. Elle reste une expérience, en sorte que seul celui qui participe en quelque manière à la vie mystique peut aussi en tirer des enseignements, et que "le mysticisme ne dit rien, absolument rien, à celui qui n'en a pas éprouvé quelque chose” (BERGSON 1932, 251). Mais alors qu'en 1903 cette expérience était celle de la découverte de l'immédiateté du soi à soi-même dans la durée, elle est ici celle de la découverte de sa propre non-originarité:

Dieu est amour, et il est objet d'amour: tout l'apport du mysticisme est là.

Amour, c'est-à-dire relation, et relation de réciprocité.

\footnotetext{
à l'intelligence (BERGSON 1907, 362). Il y aurait une étude à faire sur la réutilisation (souvent inversion), en 1932 et pour penser le rapport entre philosophie et mystique, de la théorie et des termes mêmes qui caractérisaient jusque là le rapport entre philosophie à la science.

${ }^{29}$ Sur ce point, voir DE BELLOY 2008.

30 "Mais l'expérience mystique a-t-elle besoin d'être éclairée du dedans par une intuition, comme l'étaient, par exemple, les données de la biologie ou de la mémoire? Elle est déjà constituée au moment où la philosophie la consulte pour promouvoir l'intuition qui atteignait la durée et le changement pur. Elle est à elle-même sa propre lumière.” (NABERT 1941, 356)
} 
L'expérience mystique découvre que le soi, même celui qui dure, est toujours second par rapport à la relation d'amour dans laquelle il se sent appelé, et de laquelle il se reçoit. Dans l'effort de l'intuition strictement philosophique, le soi reste toujours malgré tout étranger à lui-même, en déficit de personnalité: le mystique découvre qu'il y a, au-delà de la durée du moi, une relation primordiale qui le constitue, et que cette relation est l'émotion d'amour qu'est Dieu luimême. En cela, il bouscule le philosophe, l'oblige à sortir de lui, et dans cette sortie, à participer à l'expérience mystique, qu'il peut ainsi connaître dans une certaine mesure ${ }^{31}$.

Cependant, cette sortie ne dure pas, et ne va pas très loin. Sans quoi, le philosophe cesserait d'en être un pour devenir lui-même mystique accompli et, vivant l'immédiateté avec Dieu, abandonnerait l'intelligence et ses questions, celles de l'existence de Dieu, de sa nature, du mal, de la survie après la mort. Or s'il y revient, c'est précisément parce qu'il n'est pas devenu tout à fait mystique, et que sans renoncer à la condition humaine (celle de l'intelligence), il a cherché un compromis, choisi l'intelligence pour propager l'émotion d'amour. Il a accepté la médiation.

Mais alors, pour lui, l'intuition philosophique n'est plus la découverte de l'immanence de l'absolu à la conscience individuelle, mais bien plutôt la découverte de l'écart entre l'absolu et sa propre conscience individuelle - écart qui n'est dépassé que chez le mystique accompli, dans cette autre intuition qu'est l'intuition mystique. L'absolu apparaît désormais au philosophe irréductiblement médiat, mal-

\footnotetext{
${ }^{31}$ Nous développons ces points dans notre étude Bergson. Mystique et philosophie, Paris, PUF, 2011.
} 
gré ou plutôt à cause de sa participation à la mystique. Du coup, apparaît chez Bergson une figure jusque là inconnue, celle d'une objectivité tout à fait irréductible à la conscience qui s'en saisit:

Certains, sans aucun doute, sont totalement fermés à l'expérience mystique, incapables d'en rien éprouver, d'en rien imaginer. Mais on rencontre également des gens pour lesquels la musique n'est qu'un bruit; et tel d'entre eux s'exprime avec la même colère, sur le même ton de rancune personnelle, au sujet des musiciens. Personne ne tirera de là un argument contre la musique. (BERGSON 1932, $261)^{32}$

Pouvait-on être fermé à l'intuition de la durée? Aucun texte de Bergson, en tout cas, ne le laissait supposer. Mais en 1932, si le rapport immédiat au réel n'est plus le propre de l'intuition philosophique, mais celui de l'intuition mystique, alors il n'est pas étonnant qu'avec la médiation, un certain risque entre en philosophie, le risque pour le philosophe de rester sourd à la voix du mystique.

Rappelons-le: toute la philosophie de Bergson, même du Bergson de 1932, n'est pas dans ces affirmations. Nous sommes ici au cœur du réacteur, dans l'enceinte de confinement qui contient certes l'apport majeur, selon son auteur lui-même, des Deux Sources, mais probablement aussi son apport le plus audacieux. Car si cet apport est avancé, comme toutes les assertions publiées de Bergson, avec une grande assurance, qui traduit l'habitude bergsonienne de ne rien publier que de longuement réfléchi, sans doute ses conséquences le sont-elles moins. Bergson n'en énonce d'ailleurs que très peu. Combien moins assurée encore

\footnotetext{
32 Voir aussi (BERGSON 1932, 67): "Nous pouvons ne pas entendre distinctement leur voix ; l'appel n'en est pas moins lancé".
} 
notre glose sur ces conséquences. Elle ne saurait ici avoir pour but de proposer une interprétation complète de l'intuition philosophique révisée dans Les Deux Sources, mais seulement de pointer les difficultés qui surgissent lors d'une lecture aussi près du texte que possible, afin d'en faire pressentir la fécondité.

Qu'aurons-nous finalement acquis? D'abord, mais $\mathrm{Na}$ bert l'avait déjà bien vu, nous aurons marqué l'impossibilité, et même le contresens, qu'il y a à lire l'ensemble du bergsonisme à partir de la théorie de l'intuition développée dans la conférence de 1903. Les deux sources ont bien constitué une radicale nouveauté. Elles ont déjoué les propres plans de Bergson. Plus exactement: ils ont été déjoués, déjoués par la mystique, puisque c'est précisément cette reconnaissance de sa non-originarité par la philosophie qui constitue le bouleversement majeur de 1932. La philosophie y a-t-elle gagné ou perdu?

Cette subordination à l'expérience chrétienne de l'intuition que la philosophie annonçait et promettait, cette humilité de la philosophie, prennent chez Bergson une singulière grandeur. Et il faut dire que, en acceptant de véhiculer une émotion créatrice, la philosophie remplit une fonction qui ne la diminue pas, si du moins elle s'oriente par là vers une intuition entièrement spirituelle. (NABERT 1941, 367)

Humilité certes, voire déception: la philosophie semble s'éloigner, en faisant le "détour de l'expérience mystique", de la voie sûre de la science. Perdant la transparence intégrale à soi ${ }^{33}$, l'intuition philosophique n'est plus susceptible d'être garantie, même par la plus rigoureuse méthode, et

\footnotetext{
${ }^{33}$ NABERT 1941, 364: "L'intuition est menacée d'une sorte de scission intérieure qui lui interdit d'être transparente à soi."
} 
quoiqu'une méthode rigoureuse en soit toujours la condition. Et en même temps, peut-être ce risque, dans l'invitation du philosophe à ne plus seulement chercher le donné en lui-même mais à se le laisser véritablement donner par le mystique, est-il aussi l'un des sens de l'ouverture.

Abstract : Had Bergson anticipated, in a 1903 paper that stated the possibility to go through the intuition of the self to the intuition of an "eternity of life", his following works, and especially his great 1932 book, The Two Sources of Morality and Religion? Yes indeed, if nothing in his philosophical intuitive method changed during the thirty years in between. Following Jean Nabert (1941), this paper wants to show that the bergsonian philosophical method changes much in 1932, forced to abandon its immediacy and discovering mediacy.

Keywords: Bergson; Intuition; immediacy; mediacy; mysticism.

\section{REFERENCE}

Les références des textes de Bergson renvoient à l'édition critique des PUF, dirigée par Frédéric Worms.

BERGSON, Henri. Essai sur les données immédiates de la conscience (1889), éd. A. Bouaniche, Paris, PUF, 2007.

. Matière et mémoire (1896), éd. C. Riquier, Paris, PUF, 2008.

. Le parallélisme psycho-physique et la métaphysique positive. In: (1901), éd. C. Riquier, Paris, PUF, 2011, p. 231-272.

. Introduction à la métaphysique. In: (1903), éd. F. Fruteau de Laclos, in La Pensée et le mouvant (1934), Paris, PUF, 2009, p. 177-227.

. L’intuition philosophique. In: (1911a), éd. 
A. Feneuil \& G. Waterlot, La Pensée et le mouvant, Paris, PUF, 2009, p. 117-142.

. La perception du changement (1911b). In: éd. A. Bouaniche, La Pensée et le mouvant (1934), Paris, PUF, 2009, p. 143-176.

. Introduction I. In: (1922a), éd. A. François, La Pensée et le mouvant (1934), Paris, PUF, 2009, p. 1-23.

- Introduction II. In: (1922b), éd. A. François, La Pensée et le mouvant (1934), Paris, PUF, 2009, p. 25-98.

. Le possible et le réel. In: (1920), éd. A. Bouaniche, La Pensée et le mouvant (1934), Paris, PUF, 2009, p. 99-116.

. L'Évolution créatrice (1907), éd. A. François, Paris, PUF, 2007.

. Les Deux Sources de la morale et de la religion, éd. F. Keck \& G. Waterlot, Paris, PUF, 2008.

BELLOY, Camille de. Le philosophe et la théologie. In: Bergson et la religion. Nouvelles perspectives sur Les Deux Sources de la morale et de la religion, Paris, PUF, 2008, p. 303-319.

CHEVALIER, Jacques. Entretiens avec Bergson, Paris, Plon, 1959.

DELEUZE, Gilles. Le bergsonisme (1966), Paris, PUF, 2004.

FENEUIL, Anthony. Bergson. Mystique et philoso- 
phie, Paris, PUF, 2011.

LAWLOR, Leonard and MOULARD, Valentine. Henri Bergson. In: The Stanford Encyclopedia of Philosophy (Spring 2012 Edition), Edward N. Zalta (ed.), forthcoming URL

http://plato.stanford.edu/archives/spr2012/entries/ bergson/.

NABERT, Jean. L'intuition bergsonienne et la conscience de Dieu. In: L'expérience intérieure de la liberté et autres essais de philosophie morale, Paris, PUF, 1994, 349-367.

RIQUIER, Camille. Archéologie de Bergson, Paris, PUF, 2009.

TROTIGNON, Pierre. L'idée de vie chez Bergson et la critique de la métaphysique, Paris, PUF, 1968.

WATERLOT, Ghislain. "Doutes sur l'humanité: du "succès unique, exceptionnel" de la vie dans L'Évolution créatrice au "succès [...] si incomplet et si précaire" dans les Deux Sources". In: Annales Bergsoniennes $I V$, dir. A. Fagot-Largeault et $\mathrm{F}$. Worms, Paris, PUF, 2008, p. 379-395.

WORMS, Frédéric. Bergson ou les deux sens de la vie, Paris, PUF, 2004. 